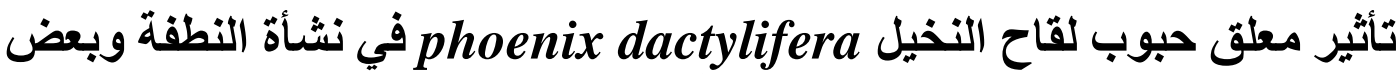 المعايير الكيموحيوية في الجرذان البيض
}

\author{
إحسان ريسان إبراهيز" إبراهيم عبيل ساجت* جبيل كريم والي" \\ استلام البحث 10، حزيران، 2010 \\ قبول النشر 26، تشرين الاول، 2010
}

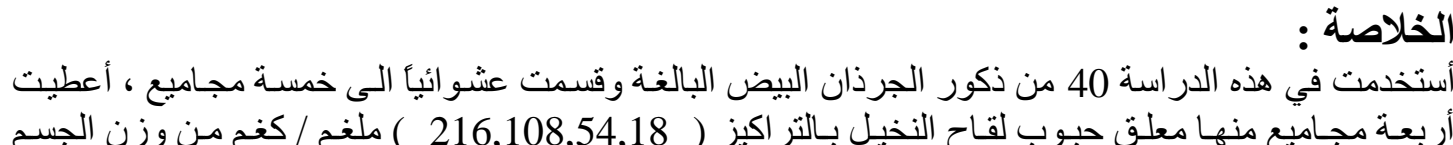

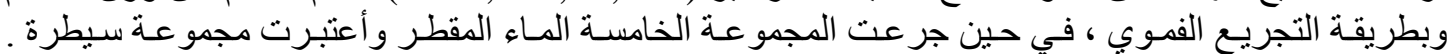

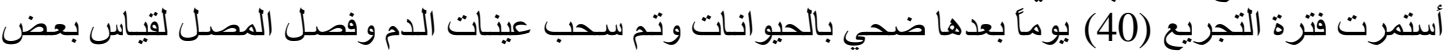

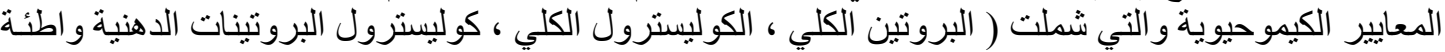

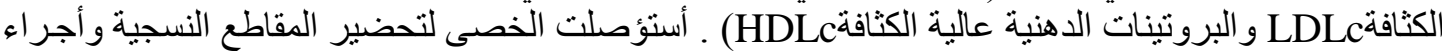

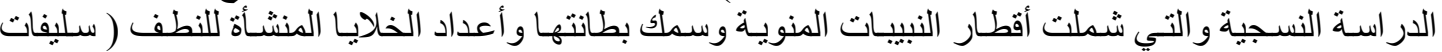

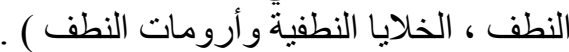

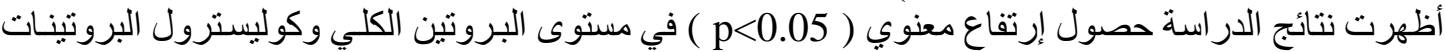

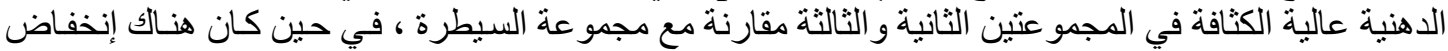

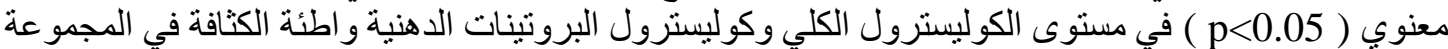

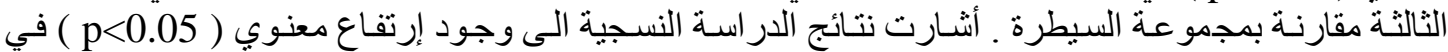

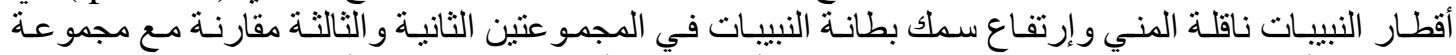

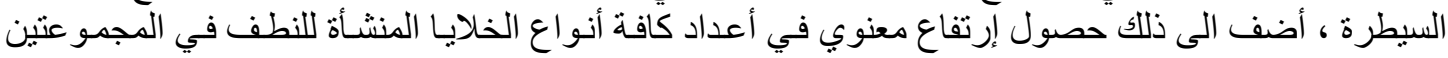

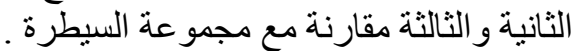

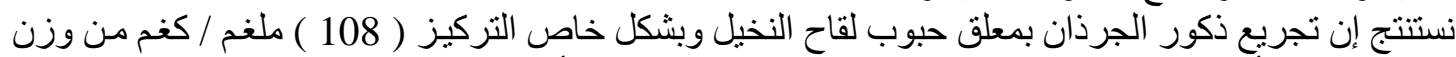

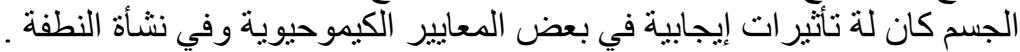

الكلمات المفتاحية:Phoenix dactylifera ،النبيبات المنوية،نشأة النطفة

Phospholipids

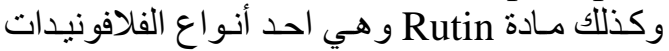

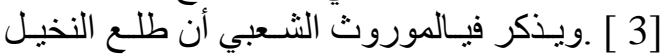

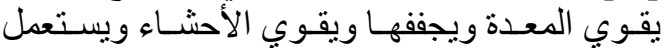

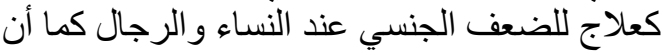

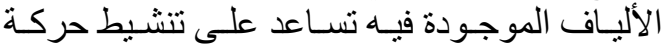

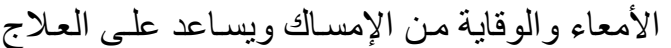

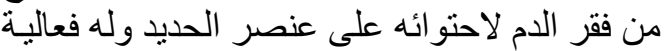

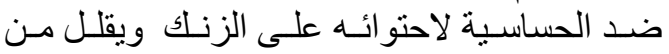

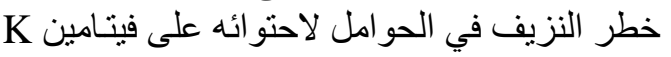

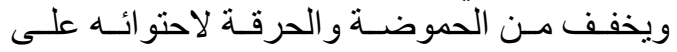

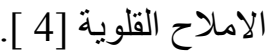

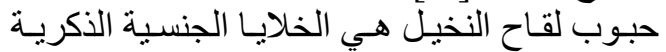

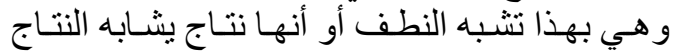

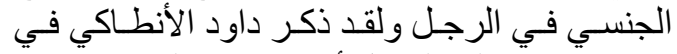

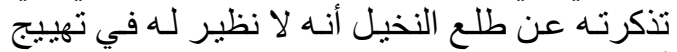

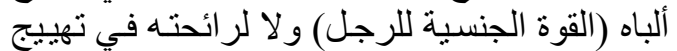

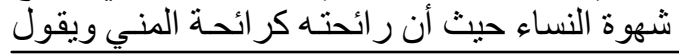

المقدمة:

كثير أ ما وصفت النخلة ومعظم أجز ائها بأنها

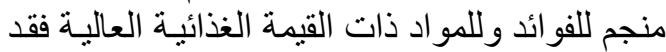

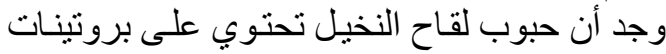
بنسبة 27.2 \% و ودهون بنسبة 20.1 \% و وسكريات بنسبة 18.1\% وكذللك تحتـوي على أليـاف بنسبة 9.9

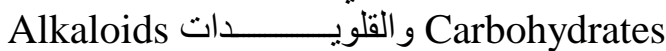

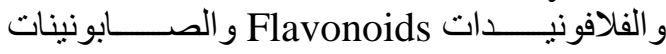

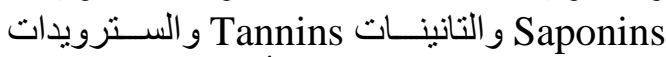

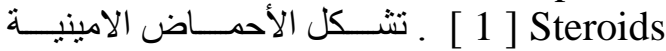

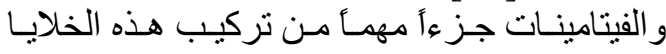

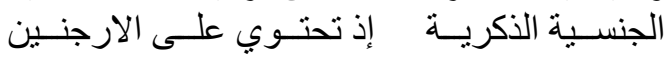

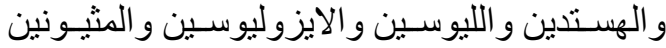

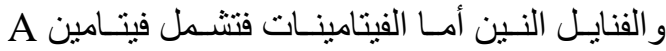

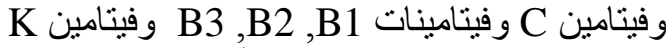

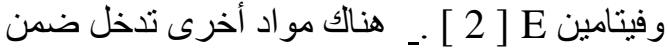
تركيب حبوب لقاح آلنخيل مثل البروتينـات السكرية

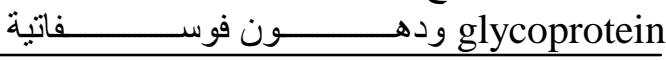
*قسم علوم الحياة / كلية التربية / جامعة القادسية 


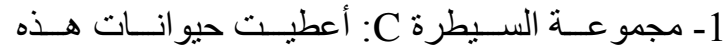
المجموعة 1 مل من الماء المقطر مرة واحة المدة يومياً.

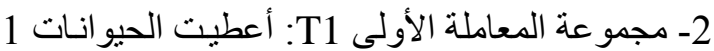

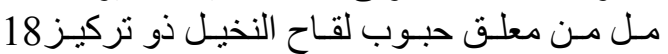
ملغم/كغم مرة واحدة يو مئاً.

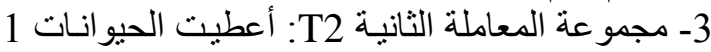

مل مـن معلـق حبـوب لقـاح النخيل ذو تركيز 54 ملغم/كغ مرة واحدة يو مياً.

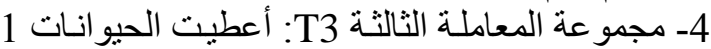
مل من معلق حبوب لقاح النخيل ذو تركيز 108 ملغم/كغم مرة و احدة يومئا.

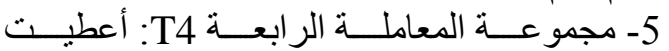
الحيو انـات 1 مل من معلق حبوب لقاح النيل النيل ذو اعطي نركيز

\section{التضحية بالحيوانات}

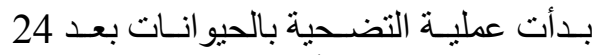
ساعة من عملية التجريع الأخيرة ، بعد سحب التحان الدام

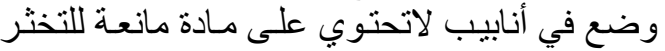

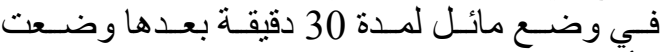
الأنابيب في جهاز الطرد المركزي لمدانئ لمدة 15 دقيقة

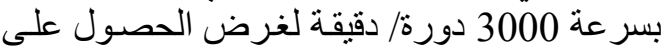

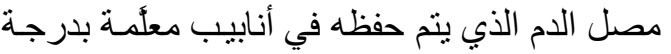

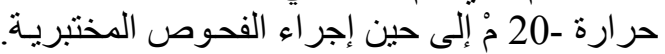

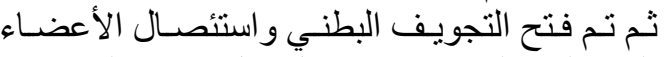

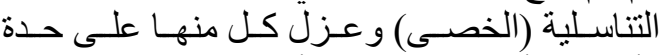
وأزيلت الأجز اء الدهنية و الأنسجة الر ابطة ثم نشفت بو اسطة ورق ترشيح حفظ الخصية في الفورمالين . \% 10

\footnotetext{
الفحوص الكيموحيوية

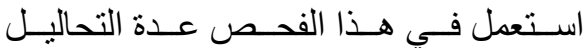

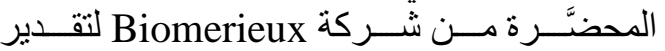

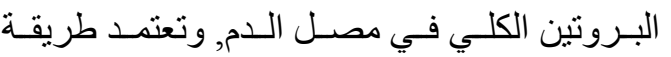

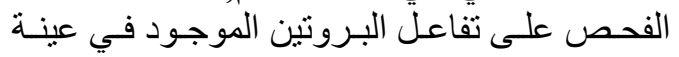

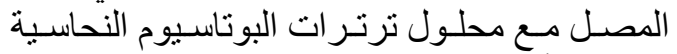
القاعديـة أو مـا يسـى بكى بكاثـف بايوريت Reagent ونتيجة هذا التفاعل يتكون معقد بنفسجي بائي اللون تعتمد شدته اللونية على على عدد أو اصر البيتيد

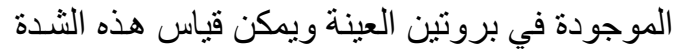

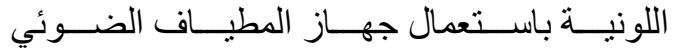
Spectrophotometer

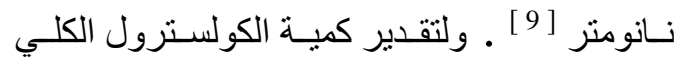
Total Cholesterol (TC) المصل,استعملت عدة القياس المجهزة مسن شـركة Biomerieux

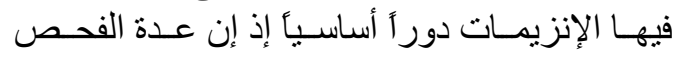
تحتوي على إنزيم Cholesterol esterase الذي دورن

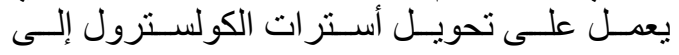

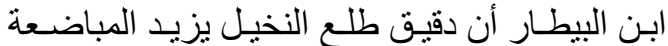

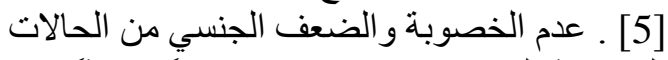

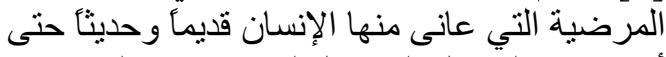

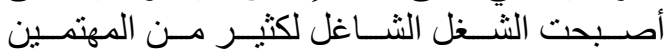

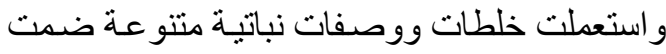

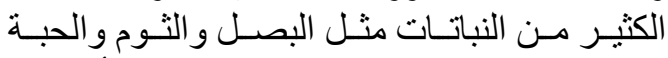

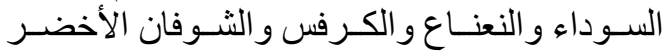

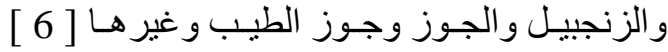
فحبوب لقاح النخيل . Phoenix dactylifera L تعتبر من أهم العلاجـات الثعبية و أقدمها, فقد كان الفيان

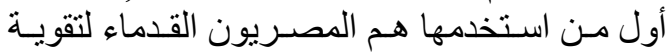

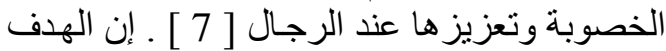

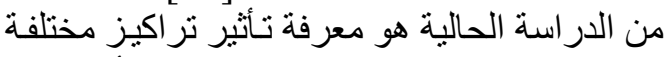

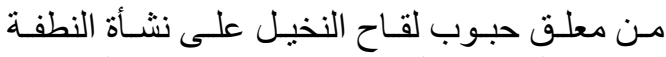

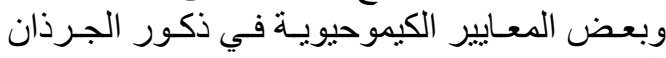

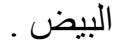

\section{المواد وطر ائق العمل : حيوانات التجربة}

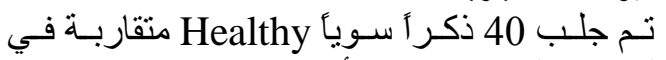
الوزن والعمر إذ كانت أوز انها تتر اوح بين(114)

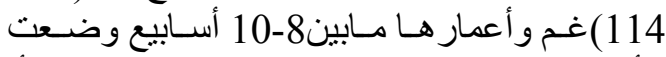

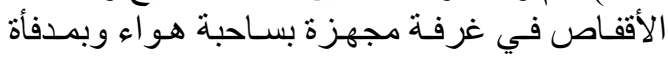

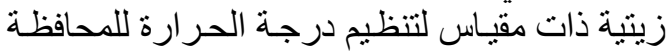

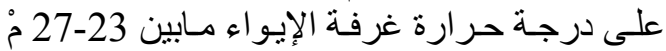

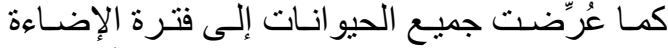

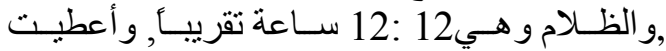

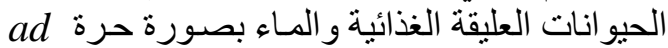

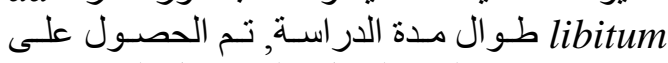

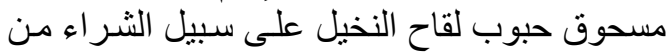

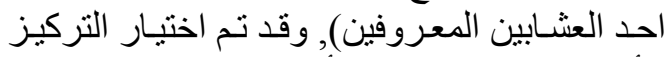

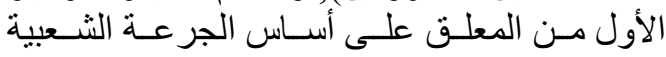

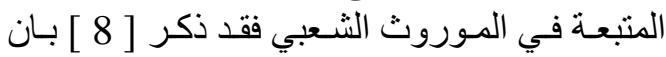

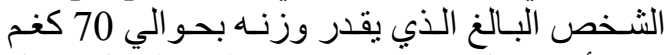
يجب أن يتناول 50 غم من حبوب لقال بـاح النخيل خـلال

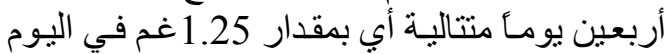

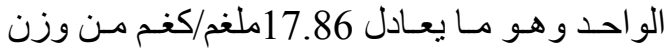

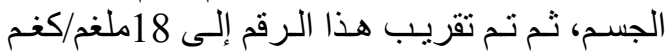

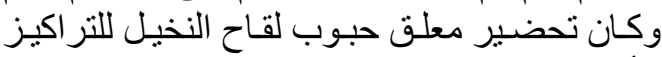
الأربعة باذابة مسحوق حبوب حيوب لقاح النخيل في المـاء

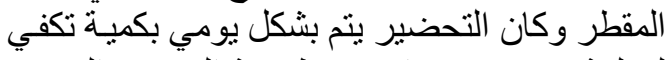

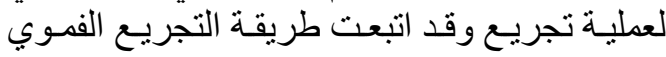
بو اسطة محقنة طبية .

قسمت حيو انات التجربة البالغ عددها أربعين

$$
\text { تصميم التجربة }
$$

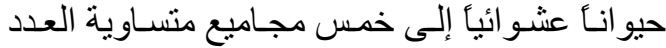

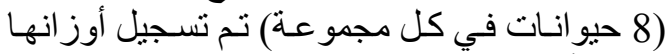

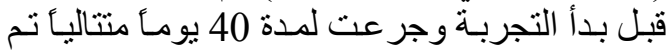
خلالها معاملة مجاميع الحيو انات على النحو الآتي: 


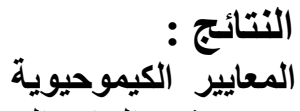

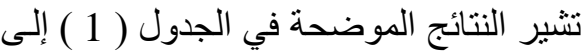

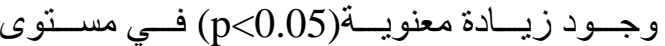
البروتين الكلي في مصل دم في معدل المجمون عندين

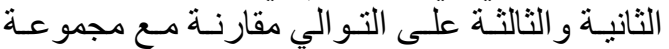
السيطرة و المجمو عتين الأولى و الر ابعة, وقد تفوقات

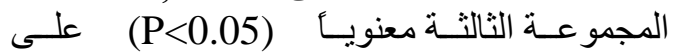

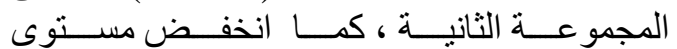
الكولسترول الكلي في مصل دم الحيو العيوانـات المعاملـة

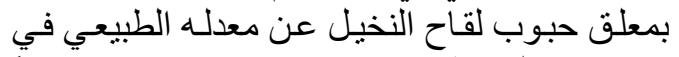

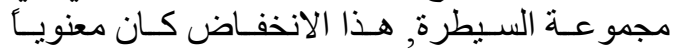
(P<0.05)

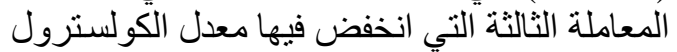

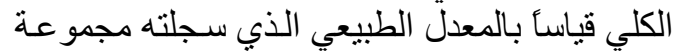

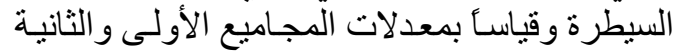

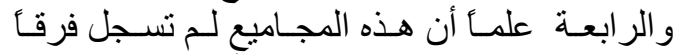

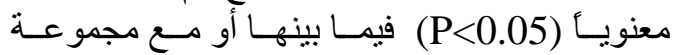

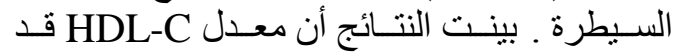

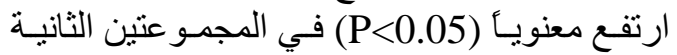

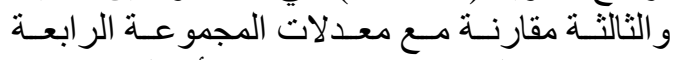

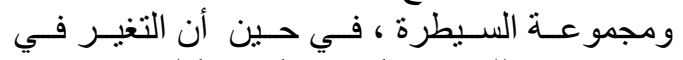

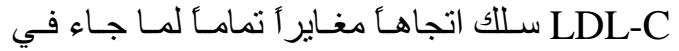

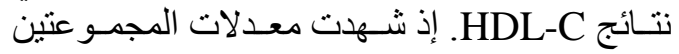

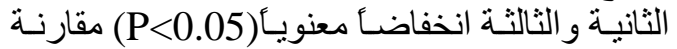
بمعدلات المجمو عة الر ابعة ومجموعة السبطرة.

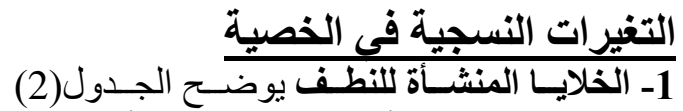

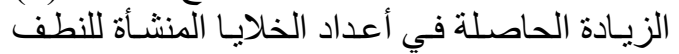
جَرَّاء التجريع بمعلق حبوب لقاح التحاح النخيل وبتر اكيز

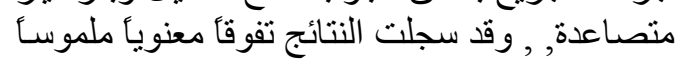

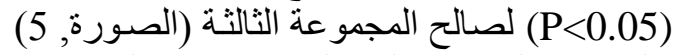

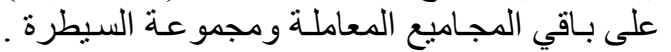

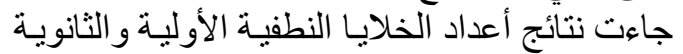

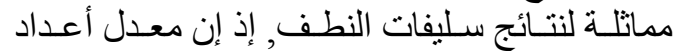

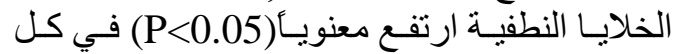

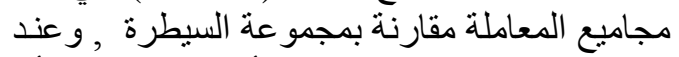

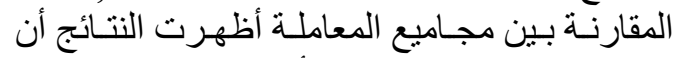

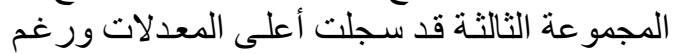
أنها لم تختلف معنوياً عن المجموعة الثنا الثانيـة إلا أنها

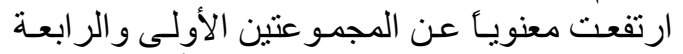

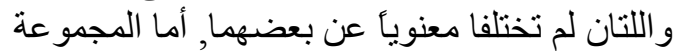

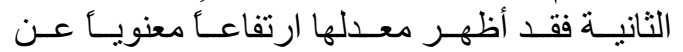
المجمو عة الر ابعة لكنه لم يسجل فرقأ معنويـاً قياسـاً

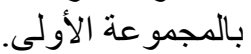
أما بالنسبة لأرومات النطف فقد بينت نتائج

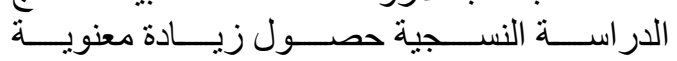

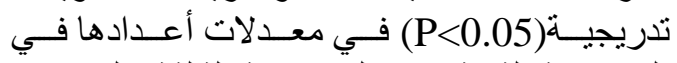
المجمو عة الثانية وفي المجموعة في الثالثة (الصورة
كولسـترول و أحمــاض دهنيـة. تـم قيـاس تركيـز

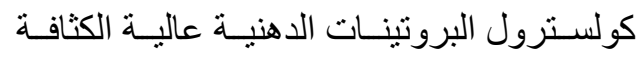
بإتباع خطو ات التعليمات المر افقة لعدة التئة (HDL-C)

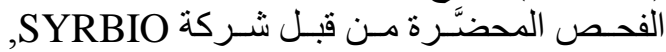

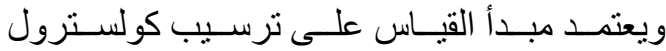

البروتينـات الدهنيـة و اطنــة الكثافــة (LDL-C) وكولسترول البروتينات الدهنية واطئة الكثافة جدأ (VLDL-C)

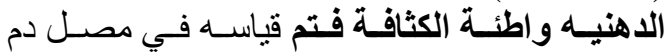
مجاميع الحيو انات باستعمال المعادلة الآتية [ 19 ] LDL-C = TC - (HDL-C + VLDL-C)

\section{تحضير المقاطع النسجية}

تم تحضير المقاطع النسجية للخصى و البر ابخ المحفوظة النية

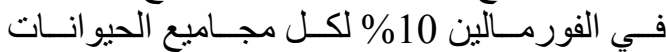

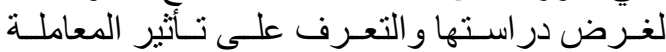

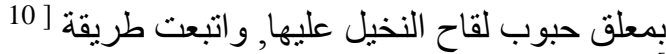

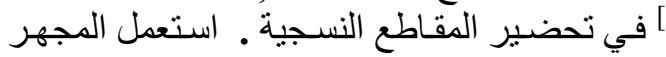

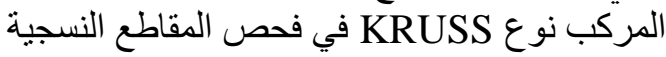
للخصية واستعمل المقياس العيني الدقيق Ocular

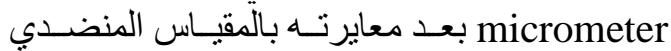

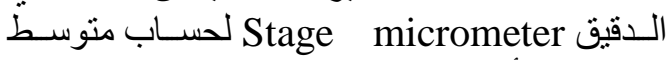

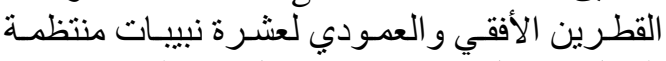

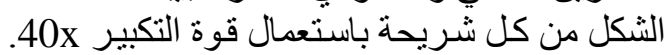

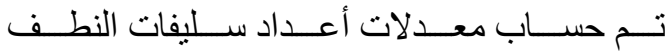
Spermatogonia

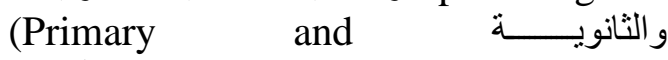
secondary)Spermatocytes Spermatids

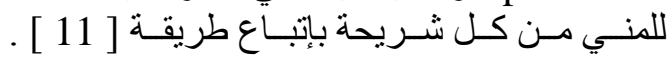

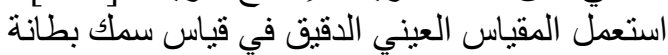

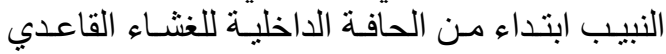

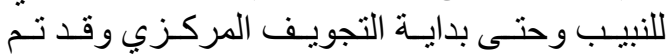
الاعتماد على معدل قر اءتين ضمن النبيب الو احد.

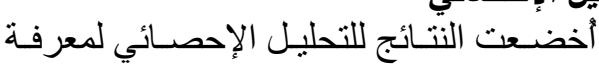

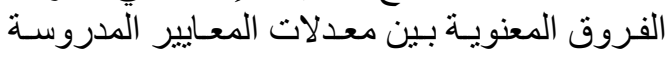

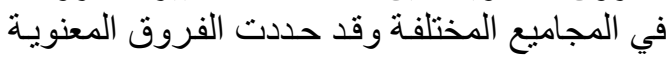

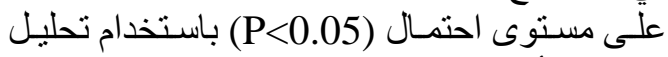
One Way Analysis of التباين الأحسادي التئي

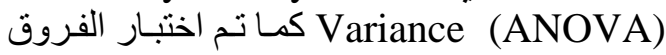
المعنوية بين المنوسطات باستخدام اختبار اقل فرق الفروق Least Significant Difference معنوين

.[12] (LSD) 
2- أقطار النبيبات ناقّة المني وسمك بطانتها

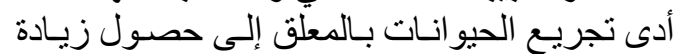

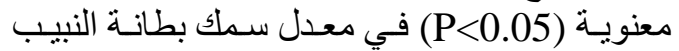

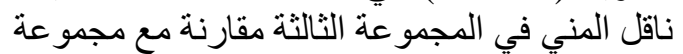

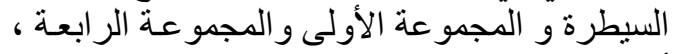

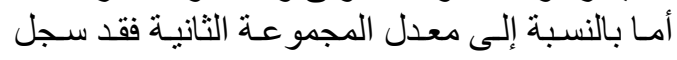

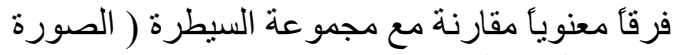

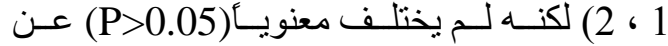
المجاميع الأولى و الثالثة و الر ابعة.

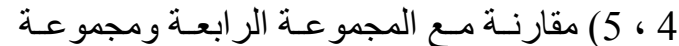

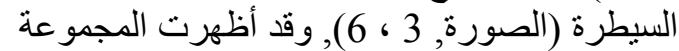

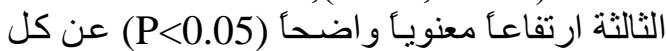

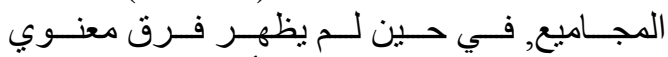
(P>0.05)

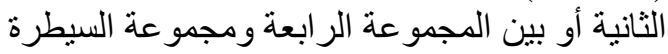

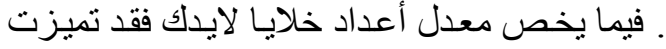

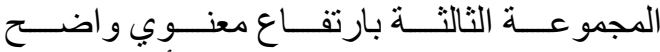
(P<0.05) مقارنة مع كل المجاميع الأخرى .

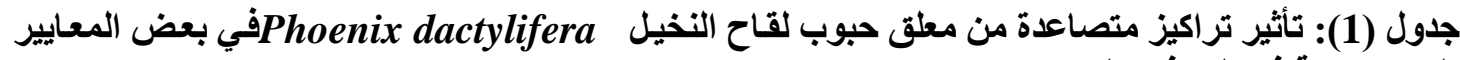

\begin{tabular}{|c|c|c|c|c|}
\hline $\begin{array}{c}\text { LDL } \\
\text { (ملفم/ ديسيلتر) }\end{array}$ & $\begin{array}{c}\text { HDL } \\
\text { (ملفر/ ديسيلتر) }\end{array}$ & (الكولسترول ديسيلتر) & (غبر/ ديسيلتر) & \\
\hline $\begin{array}{c}1.18 \pm 41.00 \\
a\end{array}$ & $\begin{array}{c}0.59 \pm 15.30 \\
\text { c }\end{array}$ & $\begin{array}{c}3.51 \pm 70.40 \\
\mathrm{a}\end{array}$ & $\begin{array}{c}0.29 \pm 4.9 \\
\text { c }\end{array}$ & $\mathbf{C}$ \\
\hline $\begin{array}{c}0.87 \pm 37.50 \\
\text { b }\end{array}$ & $\begin{array}{c}0.76 \pm 16.80 \\
\text { bc }\end{array}$ & $\begin{array}{c}2.7 \pm 68.50 \\
\mathrm{a}\end{array}$ & $\begin{array}{c}0.28 \pm 5.5 \\
c\end{array}$ & T1 \\
\hline $\begin{array}{c}\mathbf{0 . 8 8} \pm 30.50 \\
\text { c }\end{array}$ & $\begin{array}{c}0.8 \pm 18.60 \\
b\end{array}$ & $\begin{array}{c}1.9 \pm 64.34 \\
a\end{array}$ & $\begin{array}{c}0.13 \pm 6.30 \\
b\end{array}$ & T2 \\
\hline $\begin{array}{c}0.49 \pm 19.00 \\
\mathrm{~d}\end{array}$ & $\begin{array}{c}0.92 \pm 21.60 \\
a \\
\end{array}$ & $\begin{array}{c}3.10 \pm 51.50 \\
\text { b } \\
\end{array}$ & $\begin{array}{c}0.33 \pm 7.80 \\
\mathrm{a} \\
\end{array}$ & T3 \\
\hline $\begin{array}{c}0.99 \pm 38.50 \\
a b\end{array}$ & $\begin{array}{c}0.60 \pm 16.00 \\
c\end{array}$ & $\begin{array}{c}2.47 \pm 67.00 \\
\mathrm{a} \\
\end{array}$ & $\begin{array}{c}0.29 \pm 5.05 \\
\text { c } \\
\end{array}$ & T4 \\
\hline
\end{tabular}

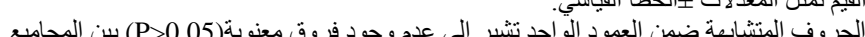
الحروف المختلفة ضمن العمن العمود الو الحد تثنير إلى إلى وجود فروق معنوية (P<0.05) بين المجاميع.

جدول (2): التأثيرات النسجية لتراكيز متصـاعدة من معلق حبوب لقاح النخيل Phoenix dactylifera في بعض صفات الخصية في الجرذان.

\begin{tabular}{|c|c|c|c|c|c|c|}
\hline أعداد خلايا لايدك & أعداد ارومات & أعداد الخلايا النظفية & أعداد سليفات & سمك بطانة النبيب ناقل & أقطار النبييات ناقلة المني & المجايير \\
\hline $\begin{array}{c}0.96 \pm 16.00 \\
\text { e }\end{array}$ & $\begin{array}{c}3.23 \pm 172.3 \\
\mathrm{e}\end{array}$ & $\begin{array}{c}2.91 \pm 101.00 \\
d\end{array}$ & $\begin{array}{c}1.73 \pm 69.00 \\
d\end{array}$ & $\begin{array}{c}4.92 \pm 89.25 \\
\mathrm{e}\end{array}$ & $\begin{array}{c}9.37 \pm 304.54 \\
\text { b }\end{array}$ & C \\
\hline $\begin{array}{c}0.81 \pm 18.00 \\
\text { be }\end{array}$ & $\begin{array}{c}2.35 \pm 184.5 \\
b\end{array}$ & $\begin{array}{c}4.97 \pm 122.00 \\
\text { be }\end{array}$ & $\begin{array}{c}1.41 \pm 84.60 \\
\text { be }\end{array}$ & $\begin{array}{l}9.03 \pm 101.87 \\
\text { be }\end{array}$ & $\begin{array}{c}8.95 \pm 312.62 \\
b\end{array}$ & T1 \\
\hline $\begin{array}{c}0.36 \pm 21.00 \\
b\end{array}$ & $\begin{array}{c}2.5 \pm 193.10 \\
b\end{array}$ & $\begin{array}{l}3.81 \pm 129.10 \\
\text { ab }\end{array}$ & $\begin{array}{c}0.85 \pm 91.20 \\
b\end{array}$ & $\begin{array}{l}5.94 \pm 109.50 \\
\text { ab }\end{array}$ & $\begin{array}{c}10.13 \pm 320.62 \\
b\end{array}$ & $\mathrm{~T} 2$ \\
\hline $\begin{array}{c}0.8 \pm 27.00 \\
a\end{array}$ & $\begin{array}{c}3.59 \pm 209.60 \\
\mathrm{a}\end{array}$ & $\begin{array}{c}3.75 \pm 137.00 \\
\text { a }\end{array}$ & $\begin{array}{c}4.1 \pm 99.80 \\
\mathrm{a}\end{array}$ & $\begin{array}{c}5.20 \pm 120.80 \\
\mathrm{a}\end{array}$ & $\begin{array}{c}11.47 \pm 362.75 \\
a\end{array}$ & T3 \\
\hline $\begin{array}{c}0.12 \pm 17.00 \\
\mathrm{e}\end{array}$ & $\begin{array}{c}38.6 \pm 175.0 \\
\mathrm{e}\end{array}$ & $\begin{array}{c}2.48 \pm 118.00 \\
\text { e }\end{array}$ & $\begin{array}{c}2.39 \pm 78.30 \\
\text { e }\end{array}$ & $\begin{array}{l}2.94 \pm 97.37 \\
\text { be }\end{array}$ & $\begin{array}{c}10.92 \pm 317.75 \\
b\end{array}$ & T4 \\
\hline
\end{tabular}

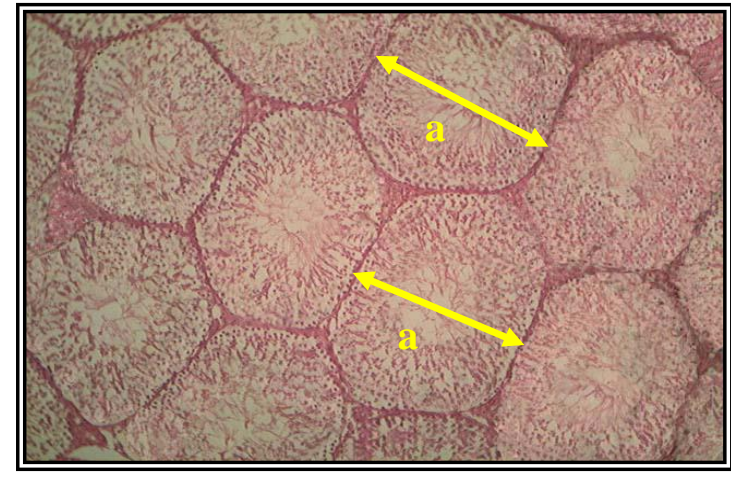

صورة (2)مقطع عرضي في الخصية تظهر فيها الزيادة

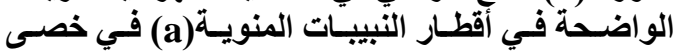
جرذان المجموعة الثالثة T3 (هيماتوكسلين_ايوسين النين (100X

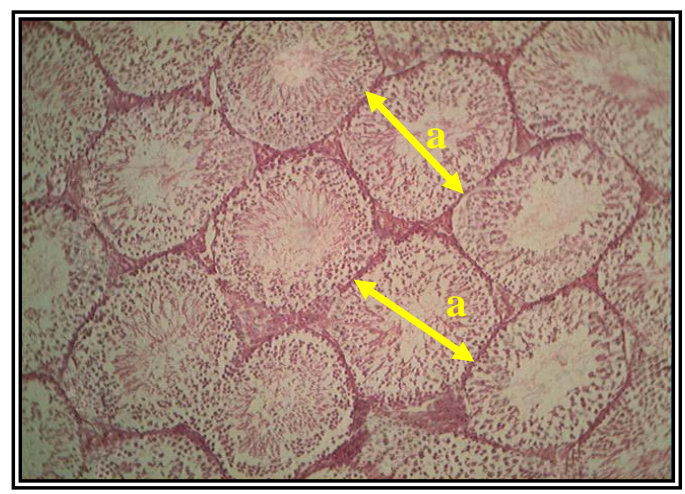

صسورة (1) مقطع في الخصية يوضـح الأقطـار

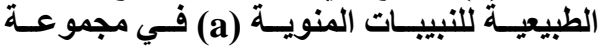
السيطرة (هيماتوكسلين_ ايوسين 100X). 


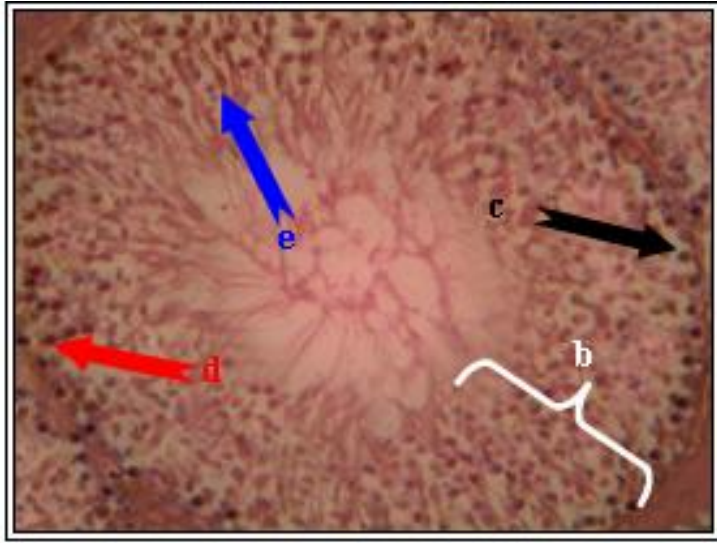

صورة(4) مقطع في التبيب المنوي لجرذ من المجموعة الثانية (T2) تظهر فيه الزيادة في سمك في بطانة التبيب

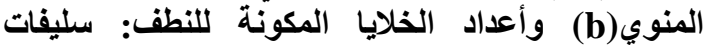

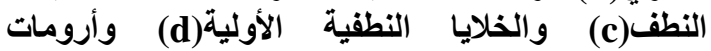
النطف(e)(هيماتوكسلين _ايوسين (c00X).

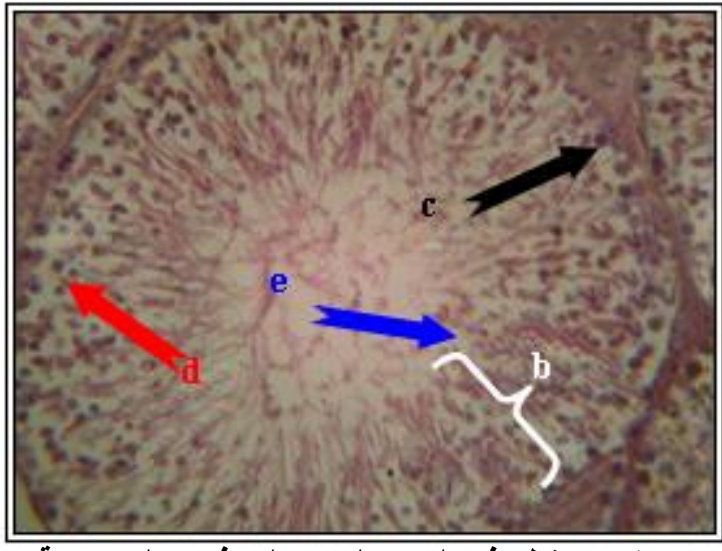

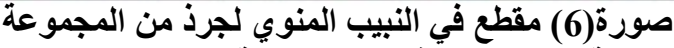

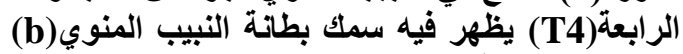

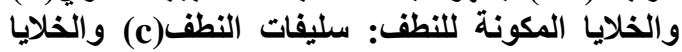

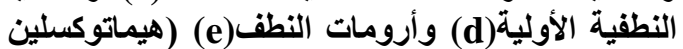
_ايوسين (400X).

البروتينات [ 14 ] قد يكون لمكونات حبوب لقاح

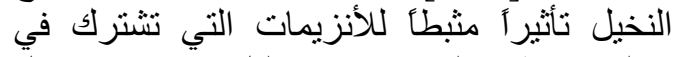
عملية تحطيم البروتينات وتقليل تركيزهاتئل داخل

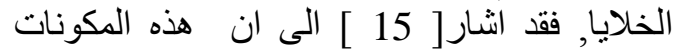
سببت ارتفاع في تركيز البروتين بسبب تأثنير ها المثبط لأنزيم Protease الهادم البروتين.

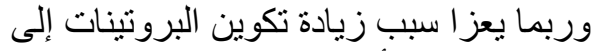

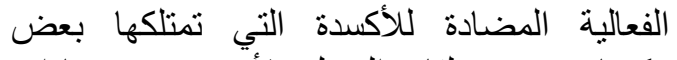
مكونات حبوب لقاح النخيل, لأن وجود الأي مضادة النات

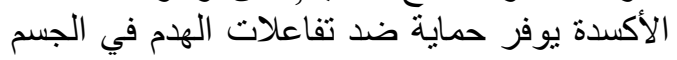

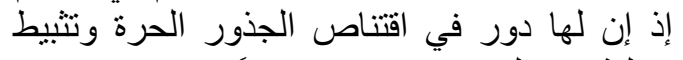

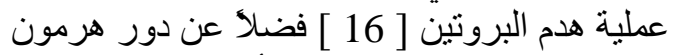
الشحمون الخصوي الذي يمكن أن بقدم تفسير آ مهماً

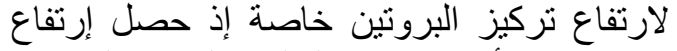

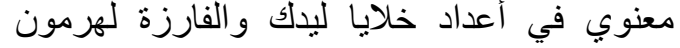

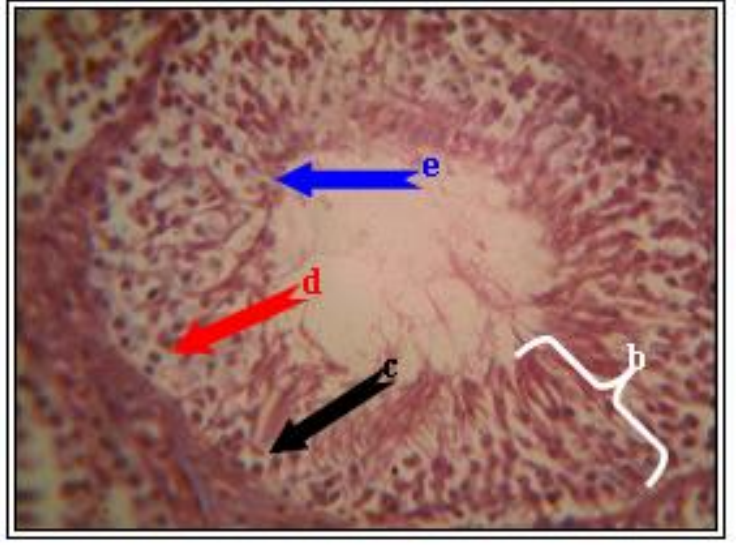

صورة(3)مقطع في النبيب المنوي لجرذ من مجموعة

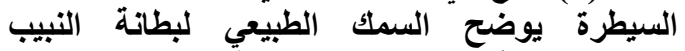

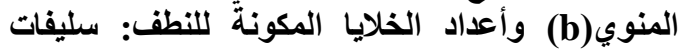
النطف(c) والخلايا النطفية الأولية(d) والنولة وأرومات

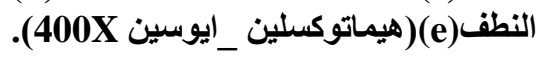

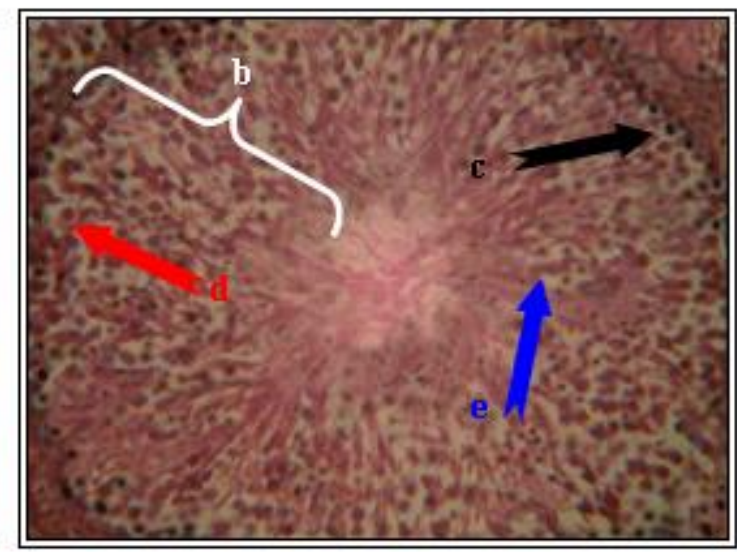

صورة(5) مقطع في النبيب المنوي لجرذ من المجموعة

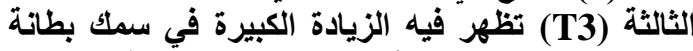

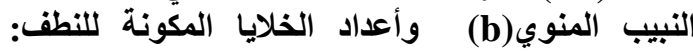

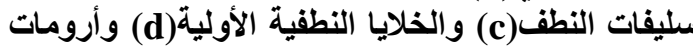

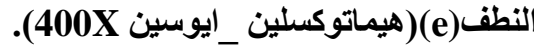

المناقشة: تأثير حبوب لقاح النخيل في المعايير الكيموحيوية

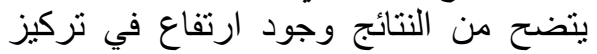
البروتين الكلي في مصل الدم في المجاميع المعاملة

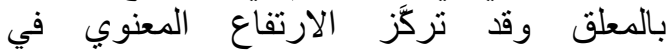

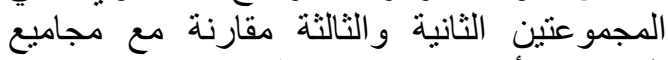

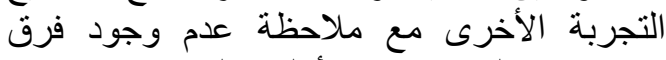

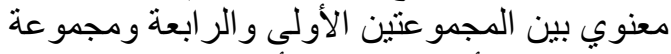
السيطرة, إن أول ما بمكن أن يتبادر إلى الفى الذهن

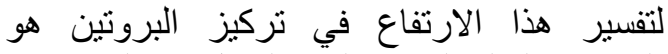
المحتوى العالي لحبوب لقاح النخيل من البرونينات

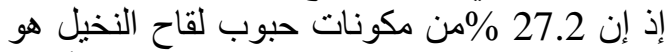
بروتين [ 13 ] وكذلك يتوفر فيها العديد من أنواع

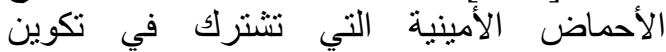


يعزى عدم تأثر المجموعة الرابعة الى عدم وجود

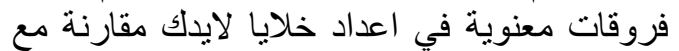

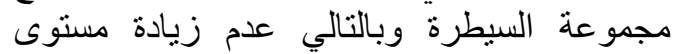

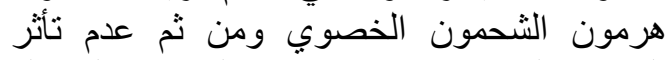
المعايير الكيموحيوية مقارنة مع المجاميع المعاملة

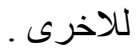

\section{التغير ات النسجية}

ان حصول زيادة في أقطار النبييات المنوية في

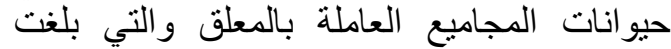
درجة المعنوية في المجموعة الثالثة مقارنة بياقي الثياتي

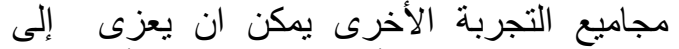

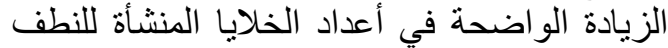

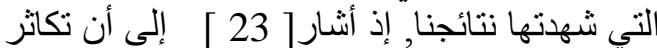

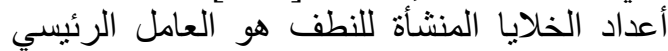

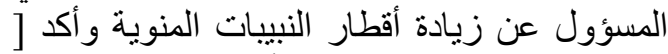

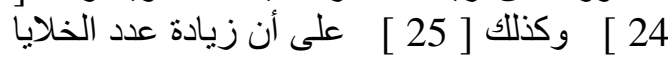
المبطنة للنبيبات المنوية ينعكس ايجابياً ـ على زيادة النهاد

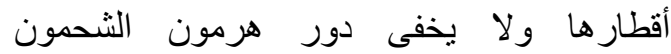
الخصوي في إدامة أقطار النبيبات المنوية وزيادة معدلات أقطار ها من خلال زئال زيادة فعالية انقسام

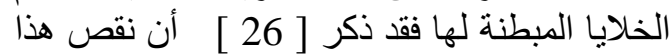

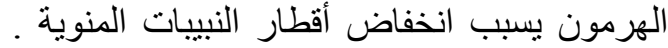

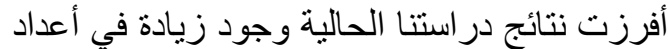
الخلايا المنشأة للنطف من سليفات نطف وخدات وخلايا

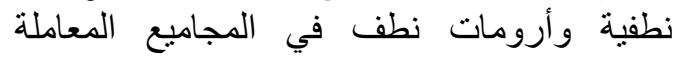

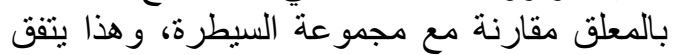

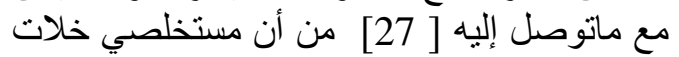
الاثثيل والكحول الاثبلي لحبوب لقاح لقاح النخيل يسبيان زيادة معنوية في أعداد سليفات النطف والئ والخلايا

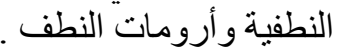

ربمـا تكون هذه الزيـادة قد نتجت مـن تأثثير معلق

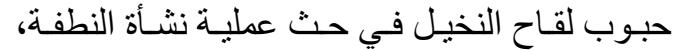

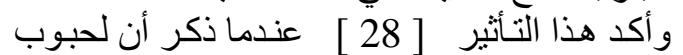

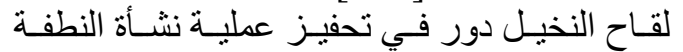

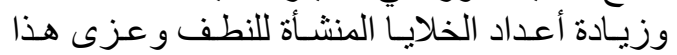

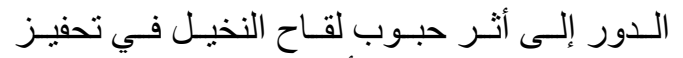

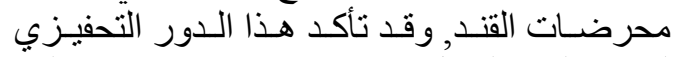

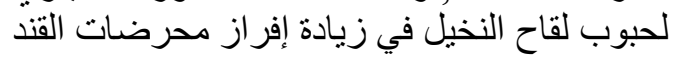

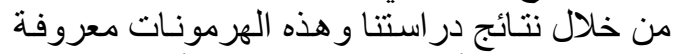
بدور ها الكبير و أثر ها المهم في زيادة أعداد العاد الخلايا

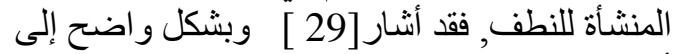

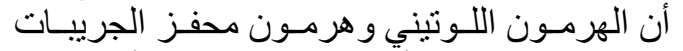

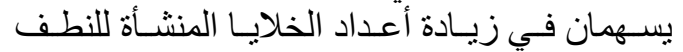

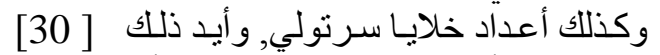

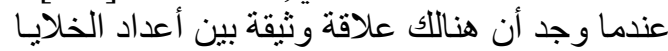

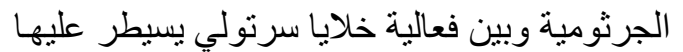

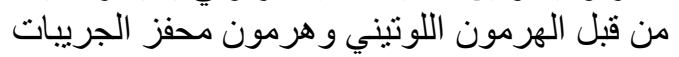

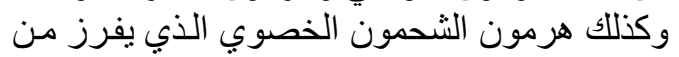

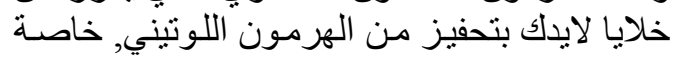

الثحمون الخصوي في المجاميع المعاملة لاسيما

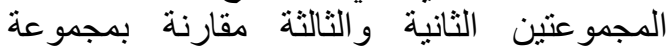

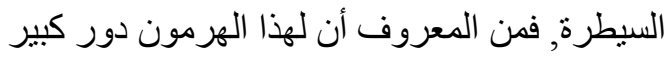
في زيادة تكوين البروتينات وتقليل عملية البرون تحطمها

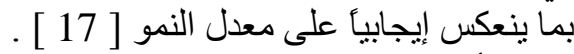

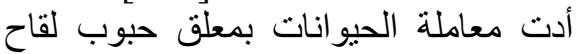
النخيل إلى انخفاض نركيز الكولسترول الكلي في مصل الدم وكذلك انخفاض تركيز التركئ كولسترول البروتينات الدهنية واطئة الكثافة(LDL-C) وكئن

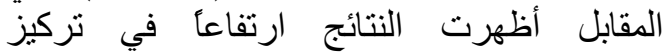

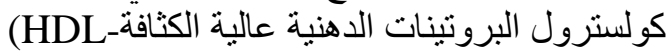
وفي مجاميع المعاملة مقارنة بمجمو عة النة السيطرة وقد اتفقت نتائج دراستنا الحالية مع النتائج التي التي

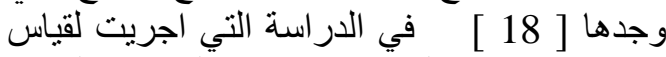

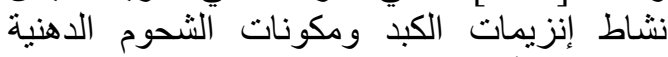
وتركيب الأحماض الدهنية في كبد ذكور الفئ الفئران

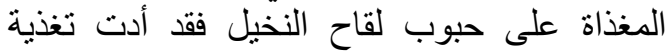

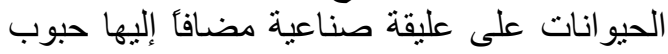

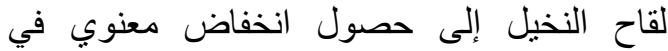
الكولسترول الكلي و الدهون الكلية وكنللك في في في في في في في بينما ظُهرت زيادة معنوية في تركيز مقارنة بالفئران المغذاة على علئ تليقة لاتحتوي عل حبوب اللقاح. يمكن أن تعدَّ هذه النتائج جميعها دليلا إضافياً

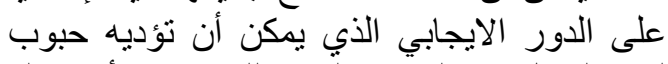

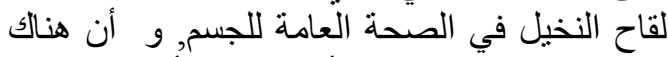

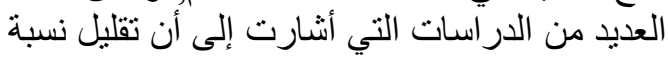

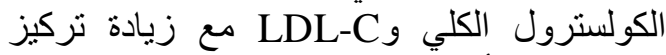

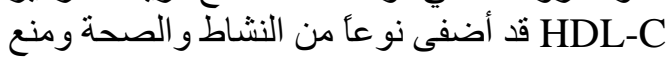
النوبات القلبية في كل من من مرضى القاطن القلب

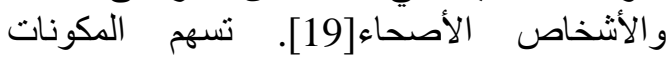
المختلفة لحبوب لقاح النخيل في هذا الانخفاض تلرئ

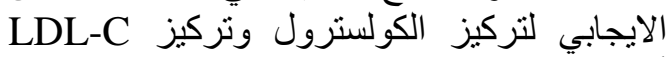

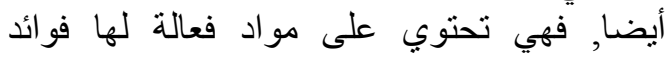

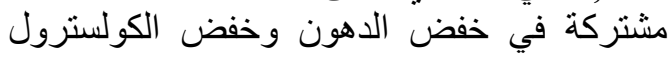

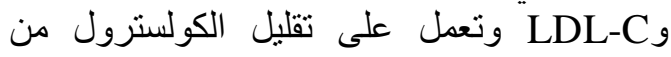
خلال التقليل من انتاج Acetyl CoA و الذي الذي يعتبر

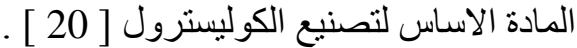

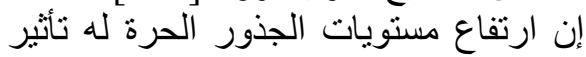

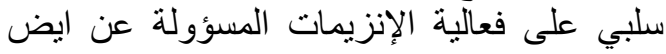
الكولَسترول في الكبد [ 21 ] ] وعليه فقد يكون الأنيد

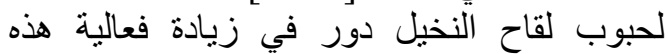

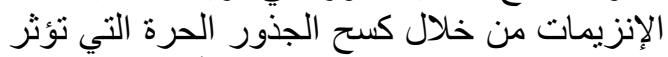

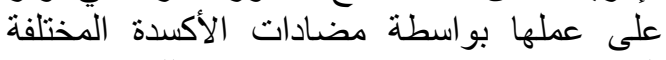

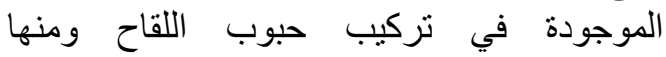

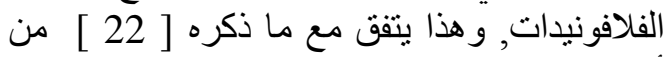

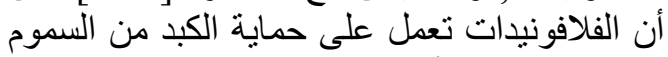

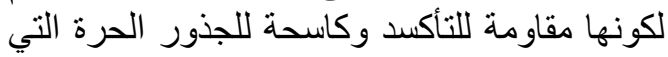

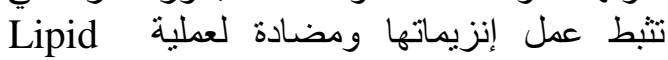
Peroxidation 
11. Al-Wachi, S. and Balash, K.J.1988. Induced alteration in spermatogenesis of mature albino mice injected with caffeine. J. Bio. Sci. Res., 19: 457-468.

12. الر اوي، خاشع محمود و خلف الله, عبد العزيز

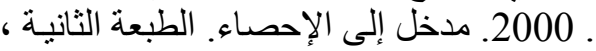
كلية الزر اعة و الغابات. جامعة الموصل.

13. Abd-Elmageed, M.M.; El- Shim, N.M. and Hassan, E.M. 1987. Sci., 15(1): 15-35.

14. Amin, A. and Sternkopf, G. 1973. On the content of amino acids in date from Iraq. Nahrnng. 17 (1): 155-257.

15. الثويني, تحريـر محمد نطـاح.2005 تـأثير

Myristica مستخلص بذور جوز الطيب

fragrans L.

البــيض وبعـض المعـايير الوظيفيـة. رســالة ماجستير . كلية العلوم-جامعة بابل.

16. Nagi, M.N.; Alam, K. and Badary, O.A. 1999. Thymoquinone protects against carbon tetrachloride hepatotoxicity in mice via an antioxidant mechanism. Biochemistry and Molecular biology International, 47(1): 153 159.

17. Saudan, C.; Baume, N.; Robinson, N.; Avois, L.; Mangin, P. and Saugy, M. 2006. Testosterone and doping control. British Journal of Sports Medicine, 40(1):21-24.

18. Al-Shagrawi, R.A. 1998. Enzyme activities, Lipid fraction and Fatty acid omposition in male rat fed Palm pollen grain (Phoenix dactylifera). Res. Bult., Agric. Res. Center, King Saud Univ., 79: 5-18.

19. Simon, H. 2006. Cholesterol, Other Lipids, and Lipoproteins .6th Avenue - DesMoines, Mercy Medical Center, p: 1-22.

20. Polanski, M.; Czarnecki, R. and Woron, J. 1996. The hepatoprotective and hypolipidemic effect of flower pollen lipid extract in androgenized rats. Folia. Med. Cracov. 37(1-2): 89-95.
وان نقص أعداد خلايـا سرتولي يؤدي إلى نقصـان في أعداد أرومات النطف بسب قلئة التجهيز الغذائي

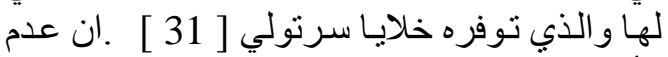

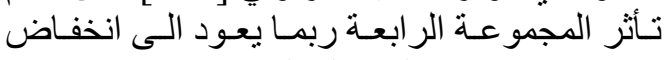

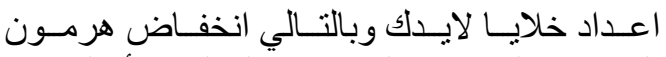
الثحمون الخصوي الضروري لعملية نشأة النطفة.

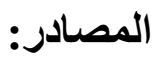

1. Bosila, H.A.; Mohamed, S.M.; ElShrabasy, S.I.; Ibrahim, A.I. and Refay, K.A. 1993. Phytochemical screening of some invivo and invitro Date Palm tissues.African J. of Biotechnology, 6(7): 410-413.

2. Amin, A. and Sternkopf, G. 1973. On the content of amino acids in date from Iraq. Nahrnng. 17 (1): 155-257.

3. Hoekstra, F.A. and Crow, J.H. 1992. Do phospholipids and Sucrose determine Membrane phase transitions in dehydration pollen species. Plant and Environment. 15(5):601-606.

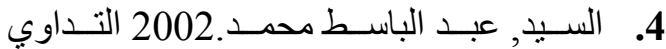

بالنبات و الطب النبوي. مطبعة مصر . مصر.

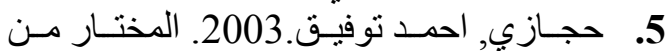

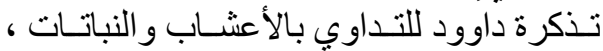

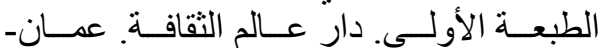

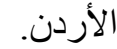

6. العبد, صبحي شـادة.2007. صيدلية النباتـات

و الأعشـاب الثشـافية. الطبعـة الأولى. دارة دار عـالم الثقافة للنشر والتوزيع. عمان- الأردن.

7. Zargari, A.1999. Medical plants. University of Tehran. Tehran press, Vol 3. P: 33-40.

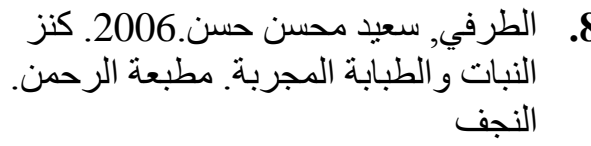

9. Burtis, C.A. and Ashwood, E.R.1999. Tietz Textbook of Clinical Chemistry $3^{\text {rd }}$ ed., W.B. Saunders Co., Philadelphia, pp: 1757-1758.

10. Luna, L.G. 1968. Manual of Histological Staining Methods of the Armed Forces Institute of Pathology. $3^{\text {rd }}$ ed. McGraw. Hill book. Co. London. 
characteristics, seminal plasma hormones and their association with testicular and epididymal histopathology. Vet. Archiv., 71: 223-236.

27. الجبوري, حسـن علـي فرمسان. 2005. تـأثير

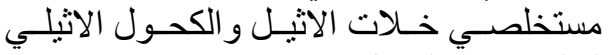

لطلع نبات النخيل Phoenix dactylifera

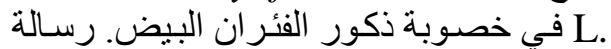

$$
\text { ماجستير . كلية العلوم-جامعة الكوفة. }
$$

28. El- Mougy, S.A.; Abel-Aziz, S.A.; Al-Shnawany, M. and Omer, A.1991. The gonadotrophic activity of Palmae in mature male rats. Alexandria J. Pharmac. Sci., 5: 156-159.

29. Orth, J.M. 1984: The role of follicle-stimulating hormone in controlling Sertoli cell proliferation in testis of fetal rats. Endocrin. 115: 1248-1255.

30. Hiller, S.G.1999. Intagonadal regulation of male and female reproduction. J. Endocrin, 60: 111117.

31. Mclachlan, R.I.; O' Donnell, L.; Meachem, S.J. and Stanon, P.G.; Dekretser, D.M.; Pratis, K. and Roberson, D.M.2002. Hormonal Regulation of spermatogenesis in primates and man: Insights for development of the male hormonal contraceptive. J. Androl., 149: 162-168.
21. Robbins, S.J.; Fasulo, J.M.; and Ordovas, J.M.2004. Diurnal changes and adaptation by the liver of hamsters to an atherogenic diet. J. Androl 24(2): 17 - 37.

22. Orisakwe, O.E.; Afonne, O.J.; Chude, M. A.; Obi, E. and Dioka, C.E.2003. Sub-chronic toxicity studies of the aqueous extract of Boerhavia diffusa leaves. J. Health Science, 49(6): 444-447.

23. Bercu, B.; Lee, B.; Pineda, L.C.; Spilotis, B.E.; Denmon, D.W. and Hoffman, H.T. 1983. Male sexual development in the Monky. A cross sectional of pulstaile hypothalamic-Pituitary-testicular function. J. Clin. Endocrinal. Metabo., 25:1214-1226.

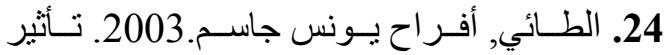

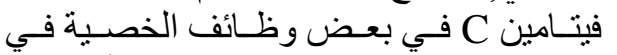

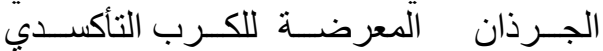

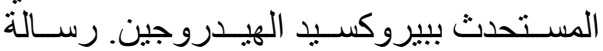

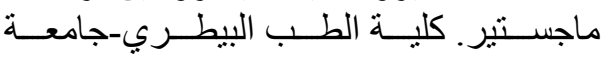
الموصل.

25. Hus, P.C.; Liu, M.Y.; Hus, C.C.; Chen, L.Y. and Guo, Y.L.1998. Effect of vitamin $\mathrm{E}$ and/or $\mathrm{C}$ on reactive oxygen species-related lead toxicity in the rat sperm. Toxicology, 128:169-179.

26. Javed, M.T.; Khan, A. and Naz, N.A.2001. Studies on abnormal buffalo Bulls with reference to scrotal circumference, semen 


\title{
Effect of Phoenix dactylifera pollen grains suspension on spermatogenesis and some biochemical parameters in albino rats
}

\author{
Ihsan R. Ibrahim* \\ Ibrahim A. Sachit* \\ Jameel. K wali*
}

*Biology Dept. / College of education/ University of AlQadisya

\begin{abstract}
:
In this study forty mature albino rats were used wich were randomly divided into five groups ,four groups were adminstrated Phoenix dactylifera pollen grains suspension at concertenrations $(18,54,108$, and 216$) \mathrm{mg} / \mathrm{kg}$ body weight by oral administration while the fifth group was considered as a control group.Experiment continued for 40 days then rats were sacrificed and samples of blood were collected for determination of some biochemical parameters (total protein ,total cholesterol ,LDLc and HDLc).Testis were removed for preparation histological sections to measures the diameters of seminferous tubules ,thickness of seminiferous epithelium and the numbers of spermatogenic cells.

Results showed significant increase $(\mathrm{p}<0.05)$ in total protein and HDLc in the second and third groups compared with a control group while there was significant decrease $(\mathrm{p}<0.05)$ in total cholesterol and LDLc in third group compared with a control group.Histological study demonstrated that there was significant increase $(\mathrm{p}<0.05)$ in diameters of seminiferous tubules and thicknnss of seminiferous epithelium in the second and third group compared with control group in addition to significant increase $(\mathrm{p}<0.05)$ in the numbers of all types of spermatogenetic cells in the second and third group compared with a control group.

It could be concluded that the adminstration of Phoenix dactylifera pollen grains suspension particulary the concentration $(108 \mathrm{mg} / \mathrm{kg}$ ) had positive effects on some biochemical parameters and spermatogenesis
\end{abstract}

